\title{
Impact of a Patient Support Program on Patient Adherence to Adalimumab and Direct Medical Costs in Crohn's Disease, Ulcerative Colitis, Rheumatoid Arthritis, Psoriasis, Psoriatic Arthritis, and Ankylosing Spondylitis
}

\author{
David T. Rubin, MD; Manish Mittal, PhD; Matthew Davis, MA; Scott Johnson, PhD, MHA; \\ Jingdong Chao, PhD; and Martha Skup, PhD
}

\begin{abstract}
BACKGROUND: AbbVie provides a free-to-patient patient support program (PSP) to assist adalimumab-treated patients with medication costs, nurse support, injection training, pen disposal, and medication reminders. The impact of these services on patient adherence to adalimumab and direct medical costs associated with autoimmune disease has not been assessed.

OBJECTIVE: To quantify the relationship between participation in a PSP and outcomes (adalimumab adherence, persistence, and direct medical costs) in patients initiating adalimumab treatment.
\end{abstract}

METHODS: A longitudinal, retrospective, cohort study was conducted using patient-level data from the PSP combined with Symphony Health Solutions administrative claims data for patients initiating adalimumab between January 2008 and June 2014. The sample included patients aged $\geq 18$ years with a diagnosis of Crohn's disease, ulcerative colitis, rheumatoid arthritis, psoriasis, psoriatic arthritis, or ankylosing spondylitis who were biologicnaïve before initiation of adalimumab. Patients who enrolled in the PSP (PSP cohort) were matched to those who did not enroll (non-PSP cohort) based on age, sex, year of treatment initiation, comorbidities, diagnosis, and initiation at a specialty pharmacy. For the PSP cohort, the index date was assigned as the earliest date of PSP enrollment, and time to enrollment following adalimumab initiation was used to assign index dates for the non-PSP cohort. All patients were required to have evidence of medical and pharmacy coverage for at least 6 months before and after their first adalimumab claim and at least 12 months after their index date. Adherence (proportion of days covered during the 12 months following PSP opt-in [index date]) was compared between cohorts using t-tests. Persistence was assessed using survival analysis of discontinuation rates. Medical costs for emergency department, inpatient, physician, and outpatient visits (all-cause and disease-related) and total costs (medical plus drug costs) were compared at 12 months following the index date using t-tests.

RESULTS: A total of 2,386 patients were included in the study and were allocated to the PSP $(n=1,199)$ and non-PSP $(n=1,187)$ cohorts. Baseline characteristics were similar between cohorts. During the follow-up period, adalimumab adherence was $14 \%$ greater in the PSP cohort than for the non-PSP cohort $(67.0 \%$ vs. $58.8 \% ; P<0.001)$. The discontinuation rate for adalimumab was $14 \%$ lower in the PSP cohort compared with the nonPSP cohort (39.7\% vs. $46.2 \%$; $P=0.001)$. Univariate analyses showed that PSP patients had $23 \%$ lower 12-month medical costs (excluding costs for biologic treatment) than did non-PSP patients $(\$ 18,322$ vs. $\$ 23,679$; $P=0.003)$. Disease-related medical costs were $22 \%$ lower for PSP than for non-PSP patients $(\$ 8,001$ vs. $\$ 10,202 ; P=0.045)$. Total costs were $10 \%$ lower for PSP than for non-PSP patients $(\$ 35,741$ vs. $\$ 39,713 ; P=0.030)$.
CONCLUSIONS: Patient enrollment in the PSP was associated with greater adherence, improved persistence, and reduced medical (all-cause and disease-related) and total health care costs for patients receiving adalimumab therapy.

J Manag Care Spec Pharm. 2017;23(8):859-67

Copyright $\odot 2017$, Academy of Managed Care Pharmacy. All rights reserved.

\section{What is already known about this subject}

Adalimumab and other currently approved biologic medications have proven clinical effectiveness against a number of autoimmune diseases, such as Crohn's disease, ulcerative colitis, rheumatoid arthritis, psoriasis, psoriatic arthritis, and ankylosing spondylitis.

Nonadherence rates to antitumor necrosis factor therapies used in these conditions exceed those observed with other medication regimens across all disease conditions.

In order to address possible risk factors for nonadherence, there is a growing trend to offer patient support programs (PSPs) to help patients and health care professionals better manage disease and optimize treatment.

\section{What this study adds}

Participation in a PSP for autoimmune diseases was associated with improved adalimumab adherence and persistence over a 12-month follow-up period.

Total cost, all-cause medical cost, and disease-related medical cost were significantly lower at 12 months in patients who participated in the PSP.

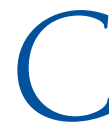

linical trials have demonstrated that antitumor necrosis factor (TNF) therapy is a safe, efficacious approach to treating inflammatory bowel disease, rheumatoid arthritis (RA), and other inflammatory diseases. ${ }^{1-5}$ In addition, real-world data suggest that anti-TNF therapy reduces the risk of extra-articular manifestations ${ }^{6}$ and that early referral to a rheumatologist leads to more timely diagnosis and treatment. ${ }^{7}$ 
However, clinical efficacy often does not fully correspond to the effectiveness encountered in clinical practice. One of the reasons for this may be that patient adherence to chronic therapy is frequently suboptimal in the real-world setting. ${ }^{8,9}$ Therefore, approaches to improve patient adherence are desirable.

Existing evidence shows that rates of nonadherence to antiTNF therapies exceed $40 \%,{ }^{10,11}$ which is greater than the average nonadherence rate of about $25 \%$ observed across all medication regimens (across all disease conditions). ${ }^{12}$ Nonadherence to prescribed therapy not only limits effectiveness but is associated with poor clinical outcomes ${ }^{13}$ and substantial economic burden. ${ }^{14}$ For example, in the use of diseasemodifying antirheumatic drugs (DMARDs) for the treatment of RA, nonadherence and discontinuation are important contributors to treatment failure, delayed recovery, accelerated progression of disease, and the need for more aggressive treatment. ${ }^{10}$ In turn, these can contribute to negative patient outcomes observable in claims data, including increased resource utilization, direct and total medical costs, and other negative consequences. ${ }^{10}$

Although no consistent risk factors for nonadherence to DMARD prescriptions have been identified, evidence suggests that patient-health care provider relationships, social support, patient beliefs about medications and about whether current health behaviors affect future health, and age contribute to medication adherence. ${ }^{15}$ In order to address possible risk factors for nonadherence, there is a growing trend to offer patient support programs (PSPs) to help patients and health care professionals better manage disease and optimize treatment, ${ }^{16}$ although no holistic analysis of a multifaceted PSP has studied its impact on patient adherence and direct medical costs in autoimmune diseases.

The manufacturer of HUMIRA (adalimumab), AbbVie, offers a unique PSP for patients across all adalimumab-approved indications. ${ }^{17}$ Components of the PSP include offering patients face-to-face interactions with a registered Nurse Ambassador; the HUMIRA Protection Plan, which offers financial assistance; provision of a container and instructions to dispose of used pens and syringes; phone, text, and/or email patient reminders to take medication; provision of materials to keep adalimumab at the required temperature for patients traveling short distances; a nurse-led injection training program; supply of resources to familiarize patients with the self-injection process; and nurse follow-up calls, in which registered nurses maintain contact with patients. Participation in the PSP is free for adalimumab patients, and all costs are covered by the manufacturer.

Evidence suggests that support such as nurse-led training programs, financial assistance, and medication reminders increase patient adherence to chronic therapies, ${ }^{18-24}$ yet a recent targeted review ${ }^{25}$ found limited studies in autoimmune disease states. One study of a general disease therapy management program for RA patients receiving injectable treatment found improvements in adherence with program participation, but it was conducted on a limited sample and study period. ${ }^{26}$ Further, there are few studies examining both adherence to therapy and health care costs. ${ }^{25}$ Therefore, this study quantified the relationship between participation in any component of the adalimumab-specific PSP on adherence and persistence as well as outcomes, including direct medical costs, in patients treated with adalimumab between 2008 and 2014 .

\section{Methods}

\section{Study Design and Data Sources}

A longitudinal, retrospective cohort study was conducted using patient-level data obtained from the PSP combined with the Symphony Health Solutions (SHS) administrative claims database. The SHS database collects longitudinal, patientlevel information on medical and pharmacy claims from a geographically diverse large set of commercial and government (Medicare and Medicaid) electronic claims processors across the United States, including International Classification of Diseases, Ninth Revision, Clinical Modification (ICD-9-CM) diagnosis codes, dates of service, charge amounts, procedure codes, National Drug Code numbers, and pharmacy types. Claims data from the SHS database were linked to the PSP data based on unique patient identifiers that were created using a series of de-identified patient identifiers (unique token) within the PSP data provider environment using a Health Insurance Portability and Accountability Act of 1996 (HIPAA)-compliant industry standard encryption engine supplied by SHS. An external HIPAA statistician evaluated the risk associated with linked data content and certified that the resulting files ensured patient anonymity.

\section{Study Population}

Patients aged $\geq 18$ years who initiated adalimumab treatment between January 2008 and June 2014 were included in this study. Only patients naive to biologic therapy before initiating adalimumab treatment (i.e., no claim for other biologic medications before the earliest adalimumab claim) were eligible for inclusion (Figure 1). A total of 3,824 patients were treated with adalimumab and met the study inclusion criteria.

Each patient was required to have $\geq 2$ claims at least 30 days apart with ICD-9-CM diagnosis codes for an autoimmune disease of interest (Crohn's disease, 555.x; ulcerative colitis, 556.x; RA, 714, 714.0, 714.8, 714.89, 714.9; psoriasis, 696, 696.1, 696.8; psoriatic arthritis, 696.0; and ankylosing spondylitis, $720,720.0$ ) before the first adalimumab use. Patients also had to have a first adalimumab claim in 2008 or later and evidence of continuous medical and drug data coverage for $\geq 6$ months before and after the first adalimumab claim, as well as $\geq 12$ months after the PSP opt-in index date (actual or imputed). 
Impact of a Patient Support Program on Patient Adherence to Adalimumab and Direct Medical Costs in Crohn's Disease, Ulcerative Colitis, Rheumatoid Arthritis, Psoriasis, Psoriatic Arthritis, and Ankylosing Spondylitis

\section{FIGURE 1 Selection of Study Population}

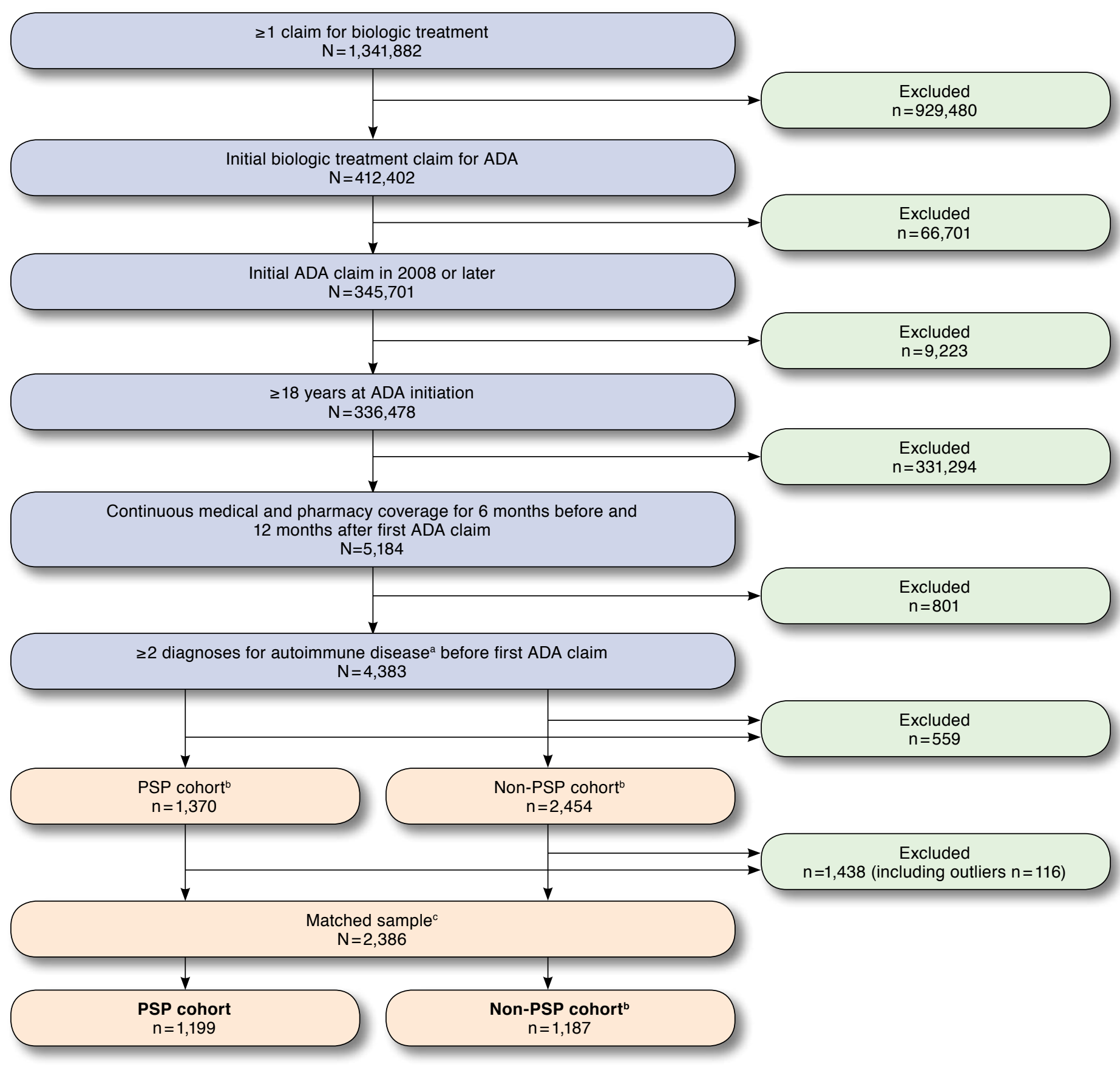

${ }^{a}$ Crohn's disease, ulcerative colitis, rheumatoid arthritis, psoriasis, psoriatic arthritis, and ankylosing spondylitis.

${ }^{b} P S P$ cohort includes patients who opted in to the PSP during or within 30 days before their first adalimumab treatment episode. Non-PSP cohort includes patients who did not opt in to the PSP during their first adalimumab treatment episode. Patients opting into the PSP more than 30 days before adalimumab initiation were excluded.

cPatients were matched 1:1 based on pharmacy type of the initial adalimumab claim and propensity score was estimated with the following covariates: age, sex, year of first adalimumab use, comorbidities, and autoimmune diagnosis. Continuous medical coverage for 12 months following the index date (PSP opt-in or imputed) was required.

$A D A=$ adalimumab; $P S P=$ patient support program. 
Impact of a Patient Support Program on Patient Adherence to Adalimumab and Direct Medical Costs in Crohn's Disease, Ulcerative Colitis, Rheumatoid Arthritis, Psoriasis, Psoriatic Arthritis, and Ankylosing Spondylitis

\section{FIGURE 2 Adherence and Persistence in Study Period}
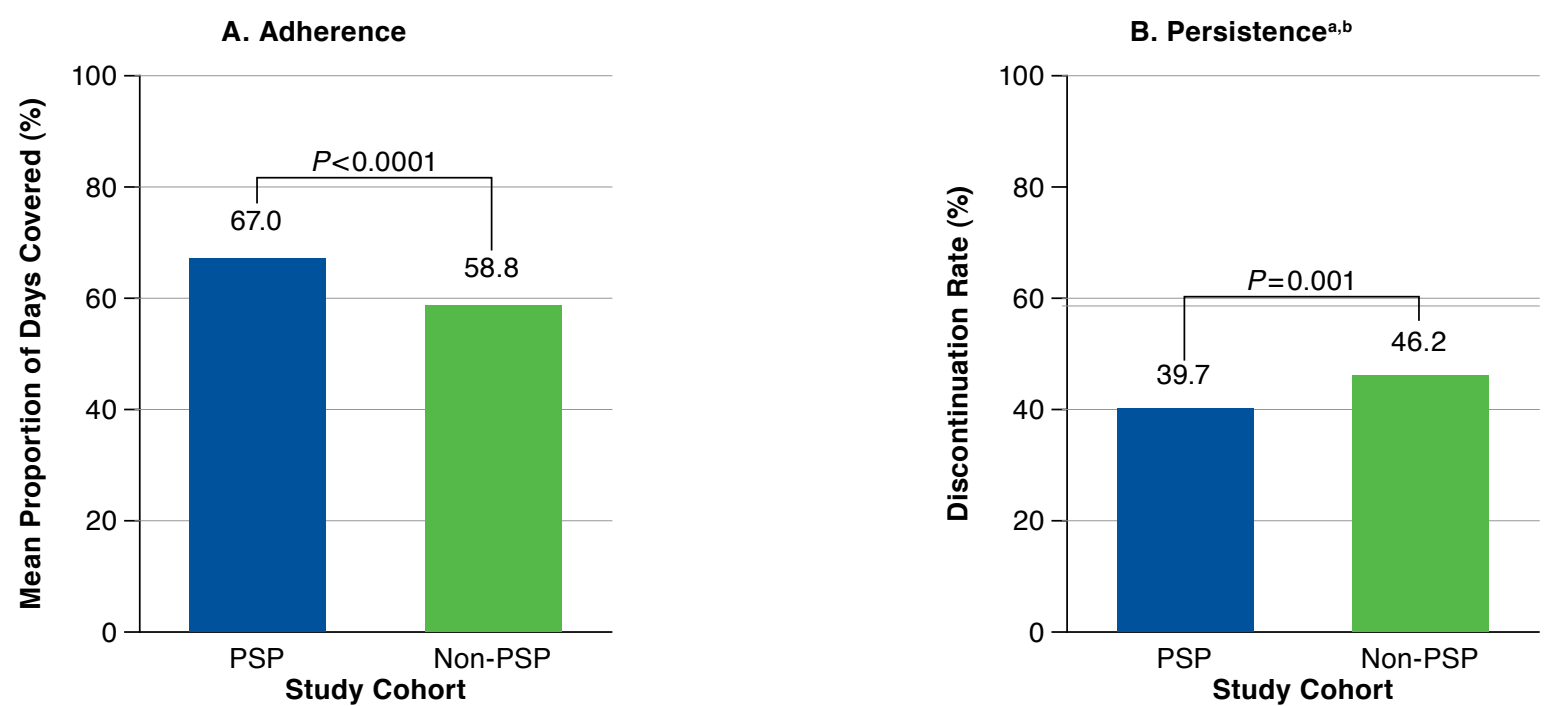

aDiscontinuation was assumed to occur if the patient switched to another biologic medication or had a gap in treatment greater than the days supply of the last adalimumab claim and never resumed the initial treatment.

${ }^{b}$ Rates and $P$ values are based on Kaplan-Meier analysis and associated log-rank test, respectively.

The SHS data do not contain an enrollment or eligibility file, so periods of continuous data coverage were required based on the observed frequency of medical and drug claims to attenuate concerns about data completeness. Algorithms to determine data coverage were developed separately for medical and drug claims by assessing gaps in consecutive claims that were predictive of potentially incomplete data. Patients were considered to have continuous medical data coverage if the interval between any 2 consecutive medical claims was no more than 120 days during the study period. Patients were considered to have continuous drug data coverage if the interval between any 2 consecutive drug dispensing records was no more than 60 days during the study period. Other studies of adherence and clinical events in these data have similarly defined periods of continuous data coverage for each patient based on the frequency of observed claims..$^{27,28}$

Patients who fulfilled the inclusion criteria were categorized into 2 cohorts according to participation in the PSP. Patients opting in to the PSP during treatment were assigned to the PSP cohort. Patients in the PSP cohort were considered to have opted in to the program if they used any of the following components of the PSP: consultation with a registered nurse, financial assistance, injection training, travel and disposal kits, and medication reminders. A patient's earliest PSP enrollment date was designated as his or her index date.

If patients did not use any of the components of the PSP, they were assigned to the non-PSP cohort. Patients in the
non-PSP cohort were matched 1:1 to patients in the PSP cohort, depending on whether their adalimumab treatment was initiated at a mail order/specialty pharmacy and using propensity scores estimated with the covariates age, sex, year of initial adalimumab claim, autoimmune disorder, and specific comorbidities.

A nearest-neighbor matching process was used in which matching was allowed with replacement to minimize differences in propensity score between cohorts. ${ }^{29}$ For each matched pair, the time from the date of adalimumab initiation to the earliest date of PSP opt-in for the PSP patient was used to assign the index date for the matched non-PSP control (e.g., if the PSP patient opted in 5 days after initiating adalimumab treatment, then the index date for the matched control was assigned to also be 5 days after initiating treatment).

\section{Outcomes}

Study outcomes included adherence to and persistence with adalimumab (defined as the proportion of days covered [PDC] and discontinuation rate, respectively) as well as direct health care cost during the 12-month follow-up period. Adherence was defined as the proportion of days with adalimumab coverage during the 12 months following PSP opt-in (index date). Patients were considered to have discontinued adalimumab if they switched to another biologic medication or had a gap in adalimumab treatment greater than the days supply on their last claim without any further adalimumab prescriptions. 


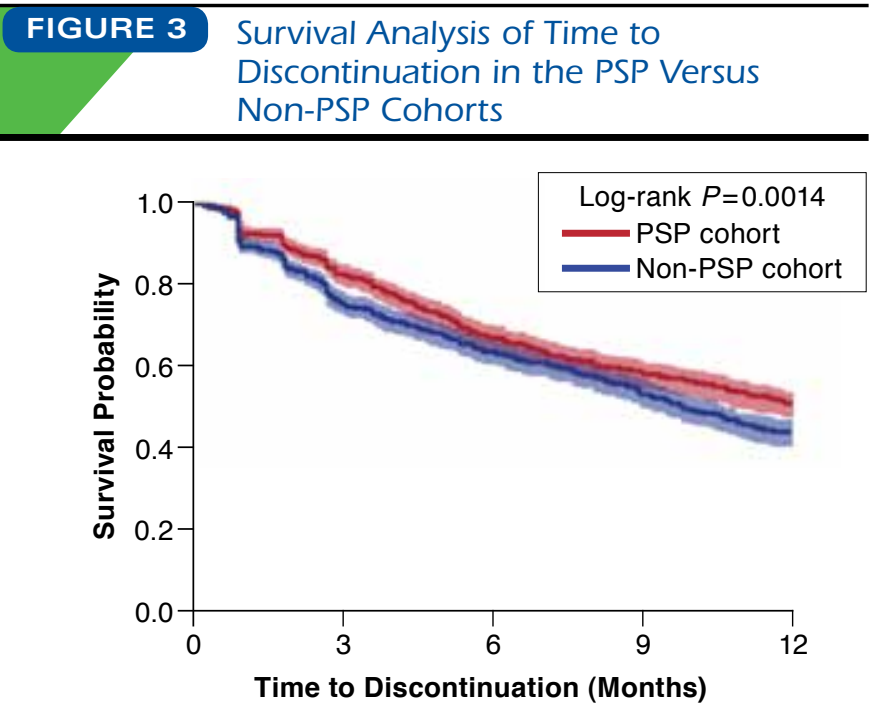

\begin{tabular}{l}
\hline Number of patients at risk \\
\begin{tabular}{l|c|r|r|r|l}
\hline PSP & 1,199 & 1,031 & 879 & 796 & 723 \\
\hline Non-PSP & 1,187 & 956 & 836 & 750 & 639 \\
\hline
\end{tabular}
\end{tabular}

PSP = patient support program.

Health care costs were also evaluated during the 12 months following the index date and included medical, drug, and total cost. Medical costs included cost associated with emergency department, inpatient, physician (i.e., medical office), and outpatient (e.g., hospital outpatient, laboratory, intermediate care facility) visits (excluding biologic costs). Drug costs included biologic costs (abatacept, adalimumab, anakinra, certolizumab pegol, etanercept, golimumab, infliximab, rituximab, and ustekinumab) covered by medical and pharmacy benefits and any other prescription cost. Total cost included medical and drug cost. Disease-related medical, drug, and total costs were also reported. Disease-related medical costs included costs from medical claims with a corresponding diagnosis of an autoimmune disease indication for each patient (excluding costs for biologics, which were considered disease-related drug costs).

The costs included in this study were captured from billing transactions between payers, hospitals, pharmacies, and physicians. For medical claims, these costs included service charges submitted to the payer. For pharmacy claims, the primary plan payment amount was included. As such, the costs in this study represent the costs to the primary payer, but they may not reflect finalized paid amounts after discounts or rebates, as discussed further in the limitations. Costs were adjusted to 2014 dollars using the Consumer Price Index for Medical Care.

\section{Statistical Analyses}

Baseline sociodemographic and clinical characteristics were summarized using descriptive statistics. Differences in baseline characteristics between the PSP and non-PSP cohorts were assessed using Student t-tests for continuous variables and chi-square tests for categorical variables. For continuous variables, Welch's t-test (or the Satterthwaite approximation) was used if the $F$ test determined variances were unequal between groups.

Adalimumab adherence and direct medical costs were compared between cohorts using t-tests. Time to adalimumab discontinuation was compared between cohorts using log-rank tests from Kaplan-Meier analysis. Patients with outlier costs, defined as costs exceeding 5 times the standard deviation of the sample, were excluded. In all analyses, a 2-sided alpha error level of 0.05 was used to indicate statistical significance. The univariate and Kaplan-Meier analyses were conducted using SAS software version 9.4 (SAS Institute, Cary, NC).

\section{Results}

\section{Sample Selection}

A total of 3,824 patients (1,370 and 2,454 in the PSP and non-PSP cohorts, respectively) were treated with adalimumab and met the study inclusion criteria (Figure 1). Following propensity-score matching and exclusion of outliers (52 and 64 patients in the PSP and non-PSP cohorts, respectively), the analysis population comprised 1,199 patients in the PSP cohort and 1,187 patients in the non-PSP cohort. Baseline characteristics were similar between the PSP and non-PSP cohorts, summarized in Table 1. The mean age of the study population was 50.8 years, and approximately $76 \%$ of patients were female. Most patients in the PSP cohort had an index date on or before the date of adalimumab initiation, with a mean time to optin of 21 days following adalimumab initiation. The majority (68\%) of patients in each cohort had an indication of RA, and the most common comorbidities were hypertension (24.1\%), hyperlipidemia (18.0\%), and diabetes mellitus type 2 (9.5\%).

\section{Adherence and Persistence}

Patients in the PSP cohort demonstrated greater adalimumab adherence and persistence than patients in the non-PSP cohort. The PSP cohort had a 14\% greater PDC (67.0\% vs. 58.8\%; $P<0.001)$ and a $14 \%$ lower discontinuation rate $(39.7 \%$ vs. $46.2 \%$; $P=0.001$ ) over the 12-month follow-up period compared with the non-PSP cohort (Figure 2). Patients in the PSP cohort also had a lower risk of discontinuation compared with patients in the non-PSP cohort $(P=0.0014$; Figure 3).

\section{Health Care Cost}

Costs were similar for PSP and non-PSP patients at baseline (Table 1). Univariate analyses of mean costs showed that PSP patients had significantly lower 12-month medical costs (excluding costs for biologics) than did non-PSP patients by $23 \%(\$ 18,322$ vs. $\$ 23,679 ; P=0.003)$. Disease-related medical costs were $22 \%$ lower for PSP than for non-PSP patients 
Impact of a Patient Support Program on Patient Adherence to Adalimumab and Direct Medical Costs in Crohn's Disease, Ulcerative Colitis, Rheumatoid Arthritis, Psoriasis, Psoriatic Arthritis, and Ankylosing Spondylitis

TABLE 1 Patient Demographic Information, Baseline Comorbidities, and Baseline Costs

\begin{tabular}{|c|c|c|c|c|c|c|c|}
\hline & PSP Cohort & $\begin{array}{l}\text { Non-PSP } \\
\text { Cohort }\end{array}$ & $\begin{array}{c}P \\
\text { Value }^{b}\end{array}$ & & PSP Cohort & $\begin{array}{l}\text { Non-PSP } \\
\text { Cohort }\end{array}$ & $\begin{array}{c}P \\
\text { Value }^{b}\end{array}$ \\
\hline Number of patients ${ }^{c}$ & 1,199 & 1,187 & - & \multicolumn{4}{|l|}{ Comorbidities, $\mathbf{n}(\%)^{\mathrm{d}}$ (continued) } \\
\hline \multirow[t]{2}{*}{ Age, years, mean \pm SD $($ median $)$} & $51.0 \pm 11.3$ & $50.6 \pm 12.9$ & 0.416 & Diabetes mellitus type 2 & $129(10.8)$ & $(8.2)$ & 0.031 \\
\hline & $(52.0)$ & $(52.0)$ & & Depression & $93 \quad(7.8)$ & $(7.2)$ & 0.580 \\
\hline \multicolumn{4}{|l|}{ Sex, n (\%) } & Sinusitis & $(5.7)$ & $(6.3)$ & 0.506 \\
\hline Male & $272(22.7)$ & $293(24.7)$ & 0.251 & Asthma & $(5.0)$ & (5.8) & 0.382 \\
\hline Female & $927(77.3)$ & $894(75.3)$ & 0.251 & \multirow{2}{*}{$\begin{array}{l}\text { Chronic obstructive } \\
\text { pulmonary disease }\end{array}$} & \multirow[t]{2}{*}{$41 \quad(3.4)$} & \multirow[t]{2}{*}{38} & \multirow[t]{2}{*}{0.766} \\
\hline \multirow{2}{*}{$\begin{array}{l}\text { Time to opt-in, days, mean } \pm \text { SD } \\
\text { (median) }\end{array}$} & $20.7 \pm 72.7$ & $20.1 \pm 76.6$ & 0.838 & & & & \\
\hline & $(0.0)$ & $(0.0)$ & & Other ischemic heart disease & (3.4) & (3.0) & 0.593 \\
\hline \multicolumn{4}{|l|}{ Indication, $\mathrm{n}(\%)^{\mathrm{d}}$} & Peripheral vascular disease & $(2.0)$ & (1.9) & 0.910 \\
\hline Rheumatoid arthritis & $818(68.2)$ & $803(67.6)$ & 0.814 & All cancers & (1.8) & $(1.7)$ & 0.901 \\
\hline Crohn's disease & $199(16.6)$ & $213 \quad(17.9)$ & 0.383 & Cerebrovascular disease & $(0.8)$ & $(0.9)$ & 0.637 \\
\hline Psoriasis & $109 \quad(9.1)$ & $115 \quad(9.7)$ & 0.613 & Chronic liver disease and cirrhosis & $(0.8)$ & $(0.2)$ & 0.036 \\
\hline Ulcerative colitis & $(7.8)$ & $(7.4)$ & 0.702 & Atherosclerosis & $(0.3)$ & $(0.7)$ & 0.240 \\
\hline Psoriatic arthritis & $(9.5)$ & $(9.1)$ & 0.738 & Congestive heart failure & $(0.6)$ & $(0.4)$ & 0.575 \\
\hline Ankylosing spondylitis & $(2.0)$ & $(1.3)$ & 0.215 & Skin cancer & $(0.4)$ & $(0.1)$ & 0.105 \\
\hline \multicolumn{4}{|l|}{ Calendar year of index date, $\mathbf{n}(\%)$} & Tuberculosis & $(0.1)$ & $(0.3)$ & 0.312 \\
\hline 2008 & $40 \quad(3.3)$ & $50 \quad(4.2)$ & 0.261 & Liver disease & $(0.2)$ & $(0.1)$ & 0.569 \\
\hline 2009 & $204(17.0)$ & $198(16.7)$ & 0.828 & Chronic renal disease & $(0.1)$ & $(0.2)$ & 0.558 \\
\hline 2010 & $222(18.5)$ & $225(19.0)$ & 0.783 & Lymphoma & $(0.1)$ & $(0.0)$ & 0.320 \\
\hline 2011 & $256(21.4)$ & $242(20.4)$ & 0.563 & Acute myocardial infarction & $(0.0)$ & $(0.0)$ & 1.000 \\
\hline 2012 & $351 \quad(29.3)$ & $359(30.2)$ & 0.604 & \multicolumn{4}{|c|}{ Baseline costs,${ }^{\mathrm{e}}$ mean \pm SD (median), $\$$} \\
\hline 2013 & $126(10.5)$ & $113 \quad(9.5)$ & 0.421 & \multirow{2}{*}{ Total costs } & \multirow{2}{*}{$\begin{array}{c}10,418 \pm 20,150 \\
(4,185)\end{array}$} & \multirow{2}{*}{$\begin{array}{c}10,147 \pm 17,216 \\
(4,481)\end{array}$} & \multirow[t]{2}{*}{0.724} \\
\hline Charlson Comorbidity Index, & $0.9 \pm 0.9$ & $0.9 \pm 0.9$ & 0.868 & & & & \\
\hline $\begin{array}{l}\text { mean } \pm \text { SD (median) } \\
\text { Comorbidities, } \mathbf{n}(\%)^{\mathrm{d}}\end{array}$ & & & & Medical costs & $\begin{array}{c}9,126 \pm 19,962 \\
(2,784)\end{array}$ & $\begin{array}{c}8,904 \pm 17,077 \\
(3,215)\end{array}$ & 0.770 \\
\hline Hypertension & $281 \quad(23.4)$ & $293(24.7)$ & 0.476 & \multirow[t]{2}{*}{ Drug costs } & \multirow{2}{*}{$\begin{array}{c}1,292 \pm 2,477 \\
(216) \\
\end{array}$} & \multirow{2}{*}{$\begin{array}{c}1,244 \pm 2,542 \\
(195) \\
\end{array}$} & \multirow{2}{*}{0.638} \\
\hline Hyperlipidemia & $220(18.3)$ & $210 \quad(17.7)$ & 0.676 & & & & \\
\hline \multicolumn{8}{|c|}{$\begin{array}{l}\text { Source: Symphony Health Solutions claims database, 2008-2014. } \\
\text { aComorbidities evaluated in the } 6 \text { months before index date. } \\
\text { bPSP cohort versus non-PSP cohort. } \\
{ }^{c} \text { Matched with } 12 \text {-month cost outliers removed. } \\
\text { dPatients may have more than } 1 \text { indication or comorbidity. } \\
\text { e Costs were evaluated in the } 6 \text { months before the index date and were calculated using the service charge for medical claims and the primary plan payment amount for } \\
\text { prescriptions. } \\
P S P=\text { patient support program: SD = standard deviation. }\end{array}$} \\
\hline
\end{tabular}

( $\$ 8,001$ vs. $\$ 10,202 ; P=0.045)$. Total costs were $10 \%$ lower for PSP than for non-PSP patients ( $\$ 35,741$ vs. $\$ 39,713 ; P=0.030$; Table 2). The main driver of lower medical costs and diseaserelated medical costs in the PSP cohort was statistically significant reductions in inpatient costs $(\$ 5,960$ vs. $\$ 10,297$, $P=0.001$, and $\$ 3,925$ vs. $\$ 5,880, P=0.041$, respectively). Differences observed in mean costs were greater than those observed using median data values. Although not statistically significant, all-cause emergency department costs and diseaserelated physician and outpatient costs also were lower for PSP than for non-PSP patients.

Consistent with increased treatment adherence and persistence, drug costs were 9\% greater ( $\$ 17,419$ vs. $\$ 16,034$; $P=0.008)$ for the PSP cohort compared with the non-PSP cohort (Table 2). Biologic drug costs were also 9\% greater for PSP than for non-PSP patients $(\$ 16,630$ vs. $\$ 15,255 ; P=0.006)$.
The main driver of greater drug and biologic costs in the PSP cohort was a statistically significant increase of $9 \%$ in adalimumab costs $(\$ 14,508$ vs. $\$ 13,264, P=0.009)$, resulting from improved adherence.

\section{Discussion}

This is one of the first studies to evaluate the impact of a multifaceted PSP for autoimmune diseases on adherence and health care costs. Enrollment in the PSP was associated with increased adalimumab adherence and persistence. In addition, mean reductions in medical costs (all-cause and diseaserelated) and total health care costs were observed in patients who enrolled in the PSP. Taken together, these findings suggest that enrollment in the PSP is associated with improved adherence to adalimumab and lower medical and overall health care cost (particularly inpatient cost). These data are of interest to 


\section{TABLE 2 Costs in Study Period ${ }^{a}$}

\begin{tabular}{|c|c|c|c|}
\hline & $\begin{array}{l}\text { PSP } \\
\text { Cohort }\end{array}$ & $\begin{array}{l}\text { Non-PSP } \\
\text { Cohort }\end{array}$ & $\begin{array}{c}P \\
\text { Value }^{\mathrm{b}} \\
\end{array}$ \\
\hline Number of patients & 1,199 & 1,187 & - \\
\hline $\begin{array}{l}\text { Total costs } \\
\text { (medical+ drug), \$ }\end{array}$ & $\begin{array}{c}35,741 \pm 38,405 \\
(27,484)\end{array}$ & $\begin{array}{c}39,713 \pm 50,072 \\
(27,740)\end{array}$ & 0.030 \\
\hline \multicolumn{4}{|l|}{ Medical costs, $\$ c$} \\
\hline Overall & $\begin{array}{c}18,322 \pm 36,792 \\
(6,151)\end{array}$ & $\begin{array}{c}23,679 \pm 49,300 \\
(7,295)\end{array}$ & 0.003 \\
\hline Emergency department & $\begin{array}{c}256 \pm 1,379 \\
(0)\end{array}$ & $\begin{array}{c}299 \pm 862 \\
(0)\end{array}$ & 0.364 \\
\hline Inpatient & $\begin{array}{c}5,960 \pm 25,144 \\
(0)\end{array}$ & $\begin{array}{c}10,297 \pm 39,067 \\
(0)\end{array}$ & 0.001 \\
\hline Physician & $\begin{array}{c}3,473 \pm 3,849 \\
(2,168)\end{array}$ & $\begin{array}{c}3,776 \pm 5,502 \\
(2,224)\end{array}$ & 0.119 \\
\hline Outpatient & $\begin{array}{c}8,633 \pm 20,096 \\
(1,940)\end{array}$ & $\begin{array}{c}9,307 \pm 21,102 \\
(2,191)\end{array}$ & 0.425 \\
\hline \multicolumn{4}{|c|}{ Disease-related medical costs, $\$$ c,d } \\
\hline Overall & $\begin{array}{c}8,001 \pm 23,336 \\
(1,530)\end{array}$ & $\begin{array}{c}10,202 \pm 29,857 \\
(1,683)\end{array}$ & 0.045 \\
\hline Emergency department & $40 \pm 495(0)$ & $36 \pm 272(0)$ & 0.793 \\
\hline Inpatient & $\begin{array}{c}3,925 \pm 19,913 \\
(0)\end{array}$ & $\begin{array}{c}5,880 \pm 26,236 \\
(0)\end{array}$ & 0.041 \\
\hline Physician & $\begin{array}{c}1,228 \pm 1,885 \\
(823)\end{array}$ & $\begin{array}{c}1,288 \pm 2,471 \\
(729)\end{array}$ & 0.503 \\
\hline Outpatient & $\begin{array}{c}2,808 \pm 10,350 \\
(0)\end{array}$ & $\begin{array}{c}2,998 \pm 9,066 \\
(71)\end{array}$ & 0.634 \\
\hline \multicolumn{4}{|l|}{ Drug costs, $\$^{\mathrm{e}}$} \\
\hline Overall & $\begin{array}{c}17,419 \pm 12,726 \\
(18,264)\end{array}$ & $\begin{array}{c}16,034 \pm 12,705 \\
(15,642)\end{array}$ & 0.008 \\
\hline Disease-related (biologics) ${ }^{\mathrm{f}}$ & $\begin{array}{c}16,630 \pm 12,292 \\
(17,680)\end{array}$ & $\begin{array}{c}15,255 \pm 12,265 \\
(15,115)\end{array}$ & 0.006 \\
\hline Adalimumab & $\begin{array}{c}14,508 \pm 11,433 \\
(14,565)\end{array}$ & $\begin{array}{c}13,264 \pm 11,754 \\
(11,345)\end{array}$ & 0.009 \\
\hline Other biologics & $\begin{array}{c}2,122 \pm 6,896 \\
(0)\end{array}$ & $\begin{array}{c}1,992 \pm 6,084 \\
(0)\end{array}$ & 0.624 \\
\hline Other prescriptions & $\begin{array}{c}789 \pm 1,752 \\
(176)\end{array}$ & $\begin{array}{c}778 \pm 1,613 \\
(181)\end{array}$ & 0.875 \\
\hline
\end{tabular}

Source: Symphony Health Solutions claims database, 2008-2014.

Note: All data are expressed as mean $\pm S D$ (median).

${ }^{a}$ Costs were evaluated over the 12-month period following the index date and were calculated using the service charge for medical claims and the primary plan payment for prescriptions.

${ }^{b} P S P$ cohort versus non-PSP cohort.

${ }^{c}$ Excludes biologic drug costs.

dIncludes costs from medical claims with a corresponding diagnosis of an autoimmune disease indication for each patient.

eIncludes biologic drugs covered by medical and pharmacy benefits, and any other prescription costs.

fIncludes etanercept, anakinra, certolizumab pegol, golimumab, ustekinumab, abatacept, infliximab, and rituximab.

$P S P=$ patient support program; $S D=$ standard deviation .

physicians, who may wish to consider selecting therapies with robust PSPs for their patients in order to achieve optimal clinical outcomes, as well as to payers, who may note the reduced costs associated with patient enrollment in a multifaceted PSP.

The PSP assessed in this study is a continuously evolving program that was designed to improve the overall patient experience with chronic inflammatory disease and its management. It provides a broad range of resources to support patients throughout their treatment with adalimumab, including frequent contact with trained health care providers. This PSP is designed to direct adalimumab patients to appropriate resources in a timely manner and provide one-on-one support to encourage adherence to therapy, including educating patients about their disease, answering medication affordability- and insurance-related questions, reinforcing injection training, and setting up medication reminders. The current results provide an early view into the impact of the PSP on increasing adalimumab adherence and decreasing health care cost to payers across a range of autoimmune disease states.

The current results are generally consistent with those of previous studies examining the impact of initiatives similar to some of the individual components of the PSP on medication adherence and health care cost. For example, electronic reminders (via mobile phone text messaging) have been shown to approximately double the odds of medication adherence in adults with chronic disease, ${ }^{30}$ and in-person, nurse-led, consultation-based interventions have been demonstrated to increase adherence to oral glucose-lowering medication in patients with type 2 diabetes. ${ }^{31}$ In addition, a national community pharmacy service, in which patients starting a new medicine for a longterm condition were supported through regular face-to-face consultations with their community pharmacists, yielded significantly improved numbers of patients adhering to their new medication. ${ }^{32}$ While of interest, these studies were not conducted for the parenteral biologic therapies, where adherence rates are typically lower, ${ }^{10,11}$ and make no link between improved adherence and reduced health care costs.

This study adds to the body of evidence examined in Ganguli et al.'s (2016) targeted systematic review of the impact of PSPs on adherence, clinical, humanistic, and economic patient outcomes. ${ }^{25}$ While comprehensive, this review reported few studies of the effect of PSPs in autoimmune disease states. The current results are in line with those of a general disease therapy management program for RA patients receiving injectable treatment, which found improvements in adherence with program participation. ${ }^{26}$ Further research examining the contributions of specific PSP components to adherence, outcomes, and cost is warranted and planned.

In addition to the novelty of the study in examining a combined set of PSP components and their impact on adherence to treatment and patient outcomes/costs, the current study has several other strengths. The administrative claims used (SHS) include a geographically diverse patient population with a broad range of health care plans, including commercial and employer plans, Medicaid, and Medicare. They also contain longitudinal, patient-level information on medical and pharmacy claims, allowing the temporal relationship between PSP enrollment and outcomes to be determined. 
The matching methodology was robust and encompassed a comprehensive variety of variables, allowing comparison between 2 cohorts of patients with similar baseline characteristics. The size of the study (more than 2,000 biologic-naive patients) and the diversity of indications that adalimumab was prescribed for across the study sample (i.e., multiple adalimumab indications) can also be considered a strength of the study. Finally, the study was conducted in a "real-world" setting, outside the controlled environment of a clinical trial, giving additional credence to the benefits demonstrated to patients (i.e., increased adherence rates and likely improved clinical outcomes) and for clinicians, administrators, and payers (i.e., lower health care costs).

\section{Limitations}

Several limitations of our study should also be noted. First, the analysis includes patients who opted into the PSP as early as 2008, that is, before some of the components of the PSP had been made available. It is expected that as increased numbers of patients opt in to PSPs, more data will be available for future studies of the newer components. Second, because the majority of patients enrolled in more than 1 component simultaneously, it was difficult to estimate the effect attributable to a single component of the PSP. The objective of this study was to examine the impact of a multifaceted, free-to-patient PSP on adherence and costs, so isolation of components of the PSP that are more or less effective was not possible. Data on the level of engagement with the PSP was not available for this study, but they will be used in future studies.

Third, individuals who chose to opt in to the PSP may be different from nonparticipating individuals in characteristics that were not observable in the data but were related to the outcomes assessed. Fourth, patients in the non-PSP cohort may have participated in assistance programs not captured in the data, such as those offered by specialty pharmacies or pharmacy benefit managers. To the extent these programs improve patient outcomes, it would make the additional estimated benefit of the adalimumab-specific PSP conservative. Fifth, the claims data used in this study did not include eligibility or health care coverage information. This meant that it could not be ensured that patients were continuously enrolled and that their complete use of medical resources covered by their payer was captured. However, this limitation was minimized by the inclusion criterion for continuous medical data coverage. Finally, charge amounts in the data may not accurately reflect final reimbursed amounts, although this is not expected to bias the relative decreases found for the PSP cohort.

\section{Conclusions}

This study of more than 2,000 patients provides the first assessment of the impact of patient enrollment in this PSP on treatment adherence and persistence and health care costs
12 months after initiation of adalimumab therapy in biologicnaive patients. Enrollment in the PSP was associated with increased adalimumab adherence and persistence, reduced medical costs (all-cause and disease-related), and reduced total health care costs. These data provide support for prescribing physicians to encourage enrollment in PSPs for inflammatory conditions and for pharmaceutical companies to further develop and invest in multifaceted PSPs.

\section{Authors}

DAVID T. RUBIN, MD, University of Chicago Medicine, Chicago, Illinois. MANISH MITTAL, PhD; JINGDONG CHAO, PhD; and MARTHA SKUP, PhD, AbbVie, North Chicago, Illinois. MATTHEW DAVIS, MA, and SCOTT JOHNSON, PhD, MHA, Medicus Economics, Milton, Massachusetts.

AUTHOR CORRESPONDENCE: David T. Rubin, MD, $5841 \mathrm{~S}$ Maryland Ave., MC 4076, Chicago, IL 60637. Tel: 773.702.2950; E-mail:drubin@medicine.bsd.uchicago.edu.

\section{DISCLOSURES}

Design, study conduct, and financial support for this study were provided by AbbVie. AbbVie participated in the interpretation of data, review, and approval of the abstract. All authors contributed to the development of the publication and maintained control over the final content. Rubin has received consulting fees or research support from AbbVie, Amgen, Emmi, Genentech, Ironwood, Janssen, Pfizer, Prometheus, Shire, and Takeda. Skup and Mittal are employees and stockholders of AbbVie. Chao was an employee of AbbVie at the time of the study and may hold AbbVie stock. Johnson and Davis are employees of Medicus Economics, which received payment from AbbVie to participate in this research

Study concept and design were contributed by Rubin, Mittal, Chao, and Skup, along with Davis and Johnson. Davis and Johnson took the lead in data collection, with assistance from the other authors, and data interpretation was performed by Rubin, Mittal, Chao, and Skup, with assistance from Davis and Johnson. All authors contributed to the writing and revision of the manuscript.

The abstract for this study was published as Rubin DT, Skup M, Davis M, Johnson S, Chao J. Impact of AbbVie's patient support program on resource costs in Crohn's disease, ulcerative colitis, rheumatoid arthritis, psoriasis, psoriatic arthritis, and ankylosing spondylitis. J Manag Care Spec Pharm. 2015;21(Suppl 4a):S74-75 (poster presentation at Academy of Managed Care, 27th Annual Meeting and Expo; April 7-10, 2015; San Diego, CA) and as abstract 2339 in Arthritis Rheumatol. 2015;67(Suppl 10; poster presentation at American College of Rheumatology 2015 ACR/AHRP Annual Meeting; November 7-11, 2015; San Francisco, CA).

\section{ACKNOWLEDGMENTS}

Medical writing support was provided by Tom Owen, PhD, of Arbor Communications, and funded by AbbVie.

\section{REFERENCES}

1. Feldmann M. Development of anti-TNF therapy for rheumatoid arthritis Nat Rev Immun. 2002;2(5):364-71. 
2. Heiberg MS, Kaufmann C, Rodevand E, et al. The comparative effectiveness of anti-TNF therapy and methotrexate in patients with psoriatic arthritis: 6 month results from a longitudinal, observational, multicentre study. Ann Rheum Dis. 2007;66(8):1038-42.

3. Maini R, St Clair EW, Breedveld F, et al. Infliximab (chimeric anti-tumour necrosis factor alpha monoclonal antibody) versus placebo in rheumatoid arthritis patients receiving concomitant methotrexate: a randomised phase III trial. ATTRACT Study Group. Lancet. 1999;354(9194):1932-39.

4. Sandborn WJ, Hanauer S, Loftus EV Jr, et al. An open-label study of the human anti-TNF monoclonal antibody adalimumab in subjects with prior loss of response or intolerance to infliximab for Crohn's disease. Am J Gastroenterol. 2004;99(10):1984-89.

5. van der Heijde D, Sieper J, Maksymowych WP, et al. 2010 Update of the international ASAS recommendations for the use of anti-TNF agents in patients with axial spondyloarthritis. Ann Rheum Dis. 2011;70(6):905-08.

6. Wendling D, Joshi A, Reilly P, Jalundhwala YJ, Mittal M, Bao Y. Comparing the risk of developing uveitis in patients initiating anti-tumor necrosis factor therapy for ankylosing spondylitis: an analysis of a large U.S. claims database. Curr Med Res Opin. 2014;30(12):2515-21.

7. Deodhar A, Mittal M, Reilly P, et al. Ankylosing spondylitis diagnosis in U.S. patients with back pain: identifying providers involved and factors associated with rheumatology referral delay. Clin Rheumat. 2016;35(7):1769-76.

8. Pincus T, Stein CM. Why randomized controlled clinical trials do not depict accurately long-term outcomes in rheumatoid arthritis: some explanations and suggestions for future studies. Clin Exp Rheumatol. 1997;15(Suppl 17): $\$ 27-38$

9. Tang B, Rahman M, Waters HC, Callegari P. Treatment persistence with adalimumab, etanercept, or infliximab in combination with methotrexate and the effects on health care costs in patients with rheumatoid arthritis. Clin Ther. 2008;30(7):1375-84.

10. Grijalva CG, Chung CP, Arbogast PG, Stein CM, Mitchel EF Jr, Griffin MR Assessment of adherence to and persistence on disease-modifying antirheumatic drugs (DMARDs) in patients with rheumatoid arthritis. Med Care. 2007:45(10 Supl 2):S66-76.

11. Yazici Y, Krasnokutsky S, Barnes JP, Hines PL, Wang J, Rosenblatt L. Changing patterns of tumor necrosis factor inhibitor use in 9074 patients with rheumatoid arthritis. J Rheumat. 2009;36(5):907-13.

12. DiMatteo MR. Variations in patients' adherence to medical recommendations: a quantitative review of 50 years of research. Med Care. 2004:42(3):200-09.

13. Bluett J, Morgan C, Thurston L, et al. Impact of inadequate adherence on response to subcutaneously administered anti-tumour necrosis factor drugs: results from the Biologics in Rheumatoid Arthritis Genetics and Genomics Study Syndicate cohort. Rheumatology (Oxford). 2015;54(3):494-99.

14. Hughes DA, Bagust A, Haycox A, Walley T. The impact of non-compliance on the cost-effectiveness of pharmaceuticals: a review of the literature. Health Econ. 2001;10(7):601-15.

15. Salt E, Frazier SK. Adherence to disease-modifying antirheumatic drugs in patients with rheumatoid arthritis: a narrative review of the literature. Orthop Nurs. 2010;29(4):260-75.

16. The ABPI Pharmacovigilance Expert Network. Guidance notes for patient safety and pharmacovigilance in patient support programmes. 2011. Available at: http://www.abpi.org.uk/our-work/library/guidelines/Pages/ patient-safety-pharmacovigilance-.aspx. Accessed February 23, 2017.
17. AbbVie. HUMIRA Complete website. Available at: https://www.humira. com/humira-complete. Accessed February 23, 2017.

18. Campbell JD, Allen-Ramey F, Sajjan SG, Maiese EM, Sullivan SD. Increasing pharmaceutical copayments: impact on asthma medication utilization and outcomes. Am J Manag Care. 2011;17(10):703-10.

19. de Jongh T, Gurol-Urganci I, Vodopivec-Jamsek V, Car J, Atun R. Mobile phone messaging for facilitating self-management of long-term illnesses. Cochrane Database Syst Rev. 2012;12:CD007459.

20. Di Battista G, Bertolotto A, Gasperini C, Ghezzi A, Maimone D, Solaro C. Multiple Sclerosis State of the Art (SMART): A Qualitative and Quantitative Analysis of Therapy's Adherence, Hospital Reliability's Perception, and Services Provided Quality. Mult Scler Int. 2014;2014:752318.

21. Kannisto KA, Koivunen MH, Valimaki MA. Use of mobile phone text message reminders in health care services: a narrative literature review. J Med Internet Res. 2014;16(10):e222.

22. Karaca-Mandic P, Joyce GF, Goldman DP, Laouri M. Cost sharing, family health care burden, and the use of specialty drugs for rheumatoid arthritis. Health Serv Res. 2010;45(5 Pt 1):1227-50.

23. Wiegand P, McCombs JS, Wang JJ. Factors of hyperlipidemia medication adherence in a nationwide health plan. Am J Manag Care. 2012;18(4):193-99.

24. Zhao RY, He XW, Shan YM, Zhu LL, Zhou Q. A stewardship intervention program for safe medication management and use of antidiabetic drugs. Clin Interv Aging. 2015;10:1201-12.

25. Ganguli A, Clewell J, Shillington AC. The impact of patient support programs on adherence, clinical, humanistic, and economic patient outcomes: a targeted systematic review. Patient Prefer Adherence. 2016;10:711-25.

26. Stockl KM, Shin JS, Lew HC, et al. Outcomes of a rheumatoid arthritis disease therapy management program focusing on medication adherence. J Manag Care Pharm. 2010;16(8):593-604. Available at: http://www.jmcp.org/ doi/abs/10.18553/jmcp.2010.16.8.593.

27. Lafeuille MH, Grittner AM, Lefebvre P, et al. Adherence patterns for abiraterone acetate and concomitant prednisone use in patients with prostate cancer. J Manag Care Spec Pharm. 2014;20(5):477-84. Available at: http:// www.jmcp.org/doi/abs/10.18553/jmcp.2014.20.5.477.

28. Ohayon MM, Black J, Lai C, Eller M, Guinta D, Bhattacharyya A. Increased mortality in narcolepsy. Sleep. 2014;37(3):439-44.

29. Stuart EA. Matching methods for causal inference: a review and a look forward. Stat Sci. 2010;25(1):1-21.

30. Elliott RA, Boyd MJ, Salema NE, et al. Supporting adherence for people starting a new medication for a long-term condition through community pharmacies: a pragmatic randomised controlled trial of the New Medicine Service. BMJ Qual Saf. 2016;25(10):747-58.

31. Farmer A, Hardeman W, Hughes D, et al. An explanatory randomised controlled trial of a nurse-led, consultation-based intervention to support patients with adherence to taking glucose lowering medication for type 2 diabetes. BMC Fam Pract. 2012;13:30.

32. Thakkar J, Kurup R, Laba TL, et al. Mobile telephone text messaging for medication adherence in chronic disease: a meta-analysis. JAMA Intern Med. 2016;176(3):340-49. 